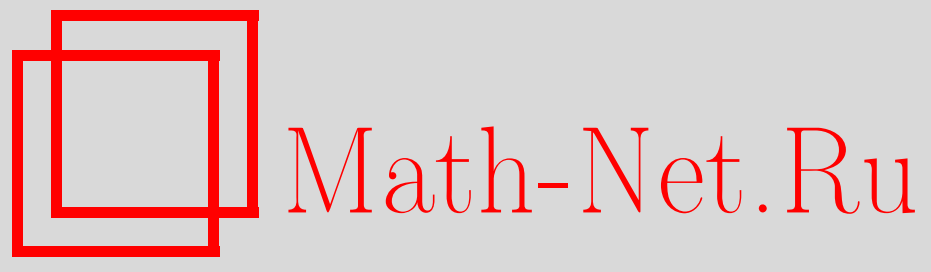

А. А. Кустарев, Циклы особенностей и характеристические классы в кобордизмах, УМН, 2007, том 62, выпуск $5,157-158$

DOI: https://doi.org/10.4213/rm7706

Использование Общероссийского математического портала Math-Net.Ru подразумевает, что вы прочитали и согласны с пользовательским соглашением http://www . mathnet.ru/rus/agreement

Параметры загрузки:

IP: 54.80 .97 .219

26 апреля 2023 г., 16:14:05

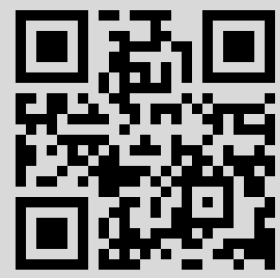




\section{Циклы особенностей и характеристические классы в кобордизмах}

\section{А. А. Кустарев}

В классической работе Л. С. Понтрягина [1] были даны два определения целочисленных характеристических классов ориентированного многообразия $M^{m}$. В одном из них рассматривались циклы точек, в которых $n \leqslant m$ общих сечений касательного расслоения $T M$ имеют ранг $k, k<n$. Во втором рассматривалось произвольное общее отображение $f: M^{m} \rightarrow \mathbb{R}^{n}$ и характеристический цикл определялся как цикл особенностей $\left[\Sigma_{f}^{r}\right], r>m-n,-$ множество точек, в которых $\operatorname{dim} \operatorname{ker} d f \geqslant r$.

С другой стороны, когомологические классы Штифеля-Уитни и Черна векторных расслоений полностью характеризуются четырьмя аксиомами (см. [2]). Как известно, существуют классы Черна в комплексных кобордизмах $c_{r}^{U}(\xi)$, удовлетворяющие этим же аксиомам [3], хотя известные конструкции их не используют методы теории особенностей.

Настоящая работа посвящена построению характеристических классов комплексных расслоений в кобордизмах при помощи циклов особенностей и исследованию их связи с классами Черна. Строятся новые геометрические реализации когомологических классов Черна $c_{r}(\xi)$ (теорема 1 ) и новые классы $P_{r}(\xi)$ и $Q_{r}(\xi)$ комплексного расслоения $\xi$ в кобордизмах. Устанавливается, что классы $P_{r}(\xi)$ и $Q_{r}(\xi)$ удовлетворяют аксиомам размерности, функториальности и нормировки, не удовлетворяют классической формуле суммы Уитни и поэтому не совпадают с $c_{r}^{U}(\xi)$ (теорема 2 и следствия).

Пусть $f: M^{2 m} \rightarrow N^{2 m-2 r+2}$ - общее отображение из комплексного многообразия $M$ в параллелизуемое комплексное многообразие $N$, дифференциал $d f$ которого комплексно-линеен, $p: \operatorname{Gr}_{r}(T M) \rightarrow M$ - каноническая проекция из $r$-й грассманизации $T M$ на $M$ и $\gamma$ - каноническое расслоение над $\operatorname{Gr}_{r}(T M)$. Тогда, согласно формуле Джамбелли-Тома-Портеуса [4], верна формула $\left[\Sigma^{r}\right]=p_{!}^{H} e\left(\operatorname{Hom}\left(\gamma, \mathbb{C}^{m-r+1}\right)\right)=$ $c_{r}(\overline{T M})$, где $p_{!}^{H}: H^{*}\left(\mathrm{Gr}_{r}(T M)\right) \rightarrow H^{*}(M)$ - гомоморфизм Гизина.

У этой формулы есть геометрическая интепретация. Если мы рассмотрим цикл $c_{r}(\overline{T M})$ как множество точек $X_{r}(\overline{T M})$, где общие $m-r+1$ сечений расслоения $\overline{T M}$ линейно зависимы, то $X_{r}(\overline{T M})$ является особым подмногообразием в $M$, особенности которого имеют вещественную коразмерность не менее двух.

Множество нулей сечения расслоения $\operatorname{Hom}\left(\gamma, \mathbb{C}^{m-r+1}\right)$ над $\operatorname{Gr}_{r}(T M)$, задаваемое дифференциалом $d f$, является неособым многообразием, разрешающим $X_{r}(\overline{T M})$. Если $X_{r}(\overline{T M})$ само неособо, то разрешение является взаимнооднозначным.

Теперь заметим, что доказательство того, что для произвольного векторного расслоения $\xi$ над $M$ верно $c_{r}(\xi)=p_{!}^{H} e\left(\operatorname{Hom}\left(\gamma, \mathbb{C}^{m-r+1}\right)\right)$, где $m=\operatorname{dim} \xi, \gamma$ - каноническое расслоение над $\operatorname{Gr}_{r}(\bar{\xi})$ и $p_{!}^{H}: H^{*}\left(\mathrm{Gr}_{r}(\bar{\xi})\right) \rightarrow H^{*}(M)$ - гомоморфизм Гизина, полностью аналогично случаю, когда $\xi=\overline{T M}$.

Таким образом, верна следующая теорема.

Теорема 1. Для произвольного расслоения $\xi$ над $M$ класс $c_{r}(\xi) \in H^{2 r}(M)$ может быть определен как $p_{!}^{H} e\left(\operatorname{Hom}\left(\gamma, \mathbb{C}^{\operatorname{dim} \xi-r+1}\right)\right)$, где $\gamma \rightarrow \operatorname{Gr}_{r}(\bar{\xi})-$ каноническое расслоение, $p$ - каноническая проекция.

Мы получаем геометрическую реализацию классов Черна комплексных векторных расслоений.

Известно другое геометрическое определение когомологических классов $c_{r}(\xi)$ [5]: если $\tau: H^{*}\left(\mathrm{Gr}_{r}(\xi)\right) \rightarrow H^{*}(M)$ - гомоморфизм трансфера [6], то $c_{r}(\xi)=\tau(e(\gamma))$.

Эти определения, как можно увидеть, обобщаются на комплексные кобордизмы $U^{*}(M)$, поскольку могут быть переформулированы с использованием только понятий класса Эйлера и гомоморфизма Гизина. В [5] доказано, что определению характеристических классов при помощи трансфера соответствуют классы $c_{r}^{U}(\xi)$ расслоения $\xi$ в комплексных кобордизмах. 
ОПредЕлЕниЕ 1 . Пусть $\xi$ - комплексное векторное расслоение размерности $m$. Определим класс $P_{r}(\xi) \in U^{2 r}$ по формуле $P_{r}(\xi)=p_{!}^{U} e\left(\operatorname{Hom}\left(\gamma, \mathbb{C}^{m-r+1}\right)\right)$, где $p_{!}^{U}$ : $U^{*}\left(\operatorname{Gr}_{r}(\bar{\xi})\right) \rightarrow U^{*}(M)$ - гомоморфизм Гизина.

Приведем конструкцию классов $Q_{r}(\xi)$ (М.Э. Казарян), схожих с классами $P_{r}(\xi)$.

Рассмотрим проективизацию $P$ тривиального комплексного $(m-r+1)$-мерного векторного расслоения над $M$, и пусть $p: P \rightarrow M-$ каноническая проекция. Тогда над $P$ возникает расслоение $\operatorname{Hom}\left(\gamma, p^{*} \xi\right)$ ( $\gamma$ - каноническое линейное расслоение над $P)$, и общие $m-r+1$ сечений $\xi$ над $M$ задают сечение $\operatorname{Hom}\left(\gamma, p^{*} \xi\right)$.

Из построения ясно, что точки, где это сечение обращается в нуль, лежат над точками цикла $X_{r}(\xi)$, причем если степень вырождения $m-r+1$ сечения равна $k>0$, то в соответствующую точку проектируется пространство $\mathbb{C} P^{k-1}$.

ОпредЕлениЕ 2. Характеристический класс $Q_{r}(\xi) \in U^{2 r}(M)$ определяется по формуле $Q_{r}(\xi)=p_{!}^{U} e\left(\operatorname{Hom}\left(\gamma, p^{*} \xi\right)\right)$, где $p_{!}^{U}: U^{*}(P) \rightarrow U^{*}(M)$ - гомоморфизм Гизина.

Теорема 2. 1. Классы $P_{r}(\xi)$ и $Q_{r}(\xi)$ функториалъны и удовлетворяют аксиомам размерности и нормировки.

2. Если иикл $X_{r}(\xi)$ является неособым, то $P_{r}(\xi)=Q_{r}(\xi)=\left[X_{r}(\xi)\right]$.

3. Для классов $P_{r}(\xi)$ и $Q_{r}(\xi)$ не выполняется формула суммы Уитни.

Первые два утверждения теоремы следуют прямо из определения. Для доказательства третьего достаточно привести пример, когда формула суммы не имеет места. Пусть $M=\mathbb{C} P^{2}, \xi=\mathscr{O}(1) \oplus \mathscr{O}(1)$. Тогда $P_{1}(\mathscr{O}(1))+P_{1}(\mathscr{O}(1))=2 t$, где $t=c_{1}^{U}(\mathscr{O}(1))$. С другой стороны, класс $P_{1}(\xi)$ совпадает с классом кобордизмов неособой кривой степени 2 в $\mathbb{C} P^{2}$, равным $2 t-\left[\mathbb{C} P^{1}\right] t^{2}$. Для класса $Q_{1}(\xi)$ доказательство аналогично.

СледСтвие 1. Если $X_{r}(\xi)$ является неособым подмногообразием в $M$, то его класс кобордизмов не обязан совпадать с $c_{r}^{U}(\xi)$.

СледСтвие 2. Классы $P_{r}(\xi)$ и $Q_{r}(\xi)$ не обязаны совпадать с $c_{r}^{U}(\xi)$.

Возникает задача - найти аналог формулы суммы Уитни для $P_{r}(\xi)$ и $Q_{r}(\xi)$, который вместе с тремя остальными аксиомами уже позволял бы установить их единственность в своем классе. Для классов $Q_{r}(\xi)$ формула может быть найдена явно.

Автор благодарен В. М. Бухштаберу за постановку задачи и внимание к работе, а также М. Э. Казаряну за ценные обсуждения.

\section{Список литературы}

[1] Л. С. Понтрягин, Матем. сб., 24(66):2 (1949), 129-162. [2] Дж. Милнор, Дж. Сташеф, Характеристические классы, Мир, М., 1979. [3] П. Коннер, Э. Флойд, Гладкие периодические отображения, Мир, М., 1969. [4] I. R. Porteous, "Simple singularities of maps", Proceedings of Liverpool singularity symposium, I (Liverpool, 1969-1970), Lecture Notes in Math., 192, ed. C. T. C. Wall, Springer, New York, 1971, 286-307. [5] В. М. Бухштабер, "Характеристические классы в кобордизмах и топологические приложения теорий однозначных и двузначных формальных групп", Современные проблемы математики, 10, ВИНИТИ, М., 1978, 5-178. [6] J. C. Becker, D. H. Gottlieb, Topology, 14:1 (1975), 1-12. [7] В. М. Бухштабер, Матем. сб., 83:4 (1970), 576-595.

\section{А. А. Кустарев (А. A. Kustarev)}

Московский государственный университет им. М. В. Ломоносова

E-mail: kustarev@gmail.com
Представлено В. М. Бухштабером Принято редколлегией 03.08.2007 